\title{
Ultra-broadband wavelength conversion based on four-wave mixing in a Raman DFB Fiber Laser
}

\author{
Jindan Shi*, Shaif-ul Alam, and Morten Ibsen \\ Optoelectronics Research Centre, University of Southampton, Highfield, Southampton, SO17 1BJ, UK \\ jxs@orc.soton.ac.uk
}

\begin{abstract}
We report highly efficient four-wave mixing (FWM) within a $30 \mathrm{~cm}$-long centre $\pi$ phase-shifted Raman DFB fiber laser with a continuous frequency tuning range of $\sim 19.9 \mathrm{THz}$ with the FWM conversion efficiency up to $-25 \mathrm{~dB}$.
\end{abstract}

(C) 2012 Optical Society of America

OCIS codes: (060.3735) Fiber Bragg gratings; (190.4380) Nonlinear optics, four-wave mixing;

\section{Introduction:}

All-optical wide-bandwidth wavelength converters are important devices for future all-optical networks [1]. Wavelength conversion utilizing four-wave mixing (FWM) has attracted considerable attention because of a number of advantages including (i) the identical features between the converted signal and its original signal, (ii) the continuous tuning range, (iii) transparency in terms of modulation format and bit-rate, and (iv) simultaneous conversion of multiple wavelength signals [2-4]. Wavelength conversion based on FWM has been demonstrated in a range of different nonlinear media; however generating it within an optical fiber is uniquely advantageous in the sense that it offers low propagation loss, long interaction length and is naturally compatible to the optical transmission systems with low insertion loss. In the conventional FWM processes in optical fibers, a strong pump and a weak probe signal are simultaneously injected into a long length of dispersion shifted fibers (DSFs), and an idler or conjugate wave can be generated when the phase-matching condition is met. Also, the bandwidth of FWM is restricted by the phase-mismatch factor, group velocity dispersion variation and the birefringence along the used DSFs [5]. Thus, in order to obtain a broadband wavelength conversion through the conventional FWM process in optical fibres, (1) the strong pump source is required to be oscillation at or near the zerodispersion wavelength (ZDW) of the DSF; and (2) the length of the DSF for FWM should be short. However, the former restricts the selection of the pump sources and the latter reduces the conversion efficiency of the FWM. Very recently, we have reported a much more compact and flexible FWM generation in a $30 \mathrm{~cm}-\mathrm{long}$ Raman DFB (R-DFB) fiber laser [6]. In that work, the DFB grating was formed in a high-NA Ge/Si fiber (UHNA4 from Nufern with a NA of 0.35) with a centre $\pi$ phase-shift. The R-DFB fiber laser at $\sim 1109.7 \mathrm{~nm}$ acted as the pump for FWM and an idler at $\sim 1158.7 \mathrm{~nm}$ was observed through the FWM between the R-DFB signal and its pump wave (at $\sim 1064.6 \mathrm{~nm}$ ). The wavelength conversion efficiency was obtained to be $-24 \mathrm{~dB}$ with a wavelength conversion range of $94.1 \mathrm{~nm}$. What is more, the R-DFB signal (1) is generated inside the RDFB cavity and consequently the coupling loss of the pump wave for FWM is nil so that the conversion efficiency can be maximised; and (2) is flexible to the ZDW of the used optical fibers. Therefore, R-DFB fiber lasers are promising FWM mixer for broadband wavelength conversion applications.

We have further investigated the FWM in the R-DFB fiber lasers incorporating a $1 \mu \mathrm{m}$ tunable laser source (TLS). In this work, we have demonstrated ultra-wide wavelength conversion by using the FWM in a $30 \mathrm{~cm}$ long R-DFB fiber laser formed in a standard NA Ge/Si optical fiber (PS980 from Fibercore Ltd.). With the integration of the TLS centred at $1060 \mathrm{~nm}$, up to $\sim 167 \mathrm{~nm}$ wavelength conversion with the conversion efficiency up to $-25 \mathrm{~dB}$ has been observed.

\section{Experimental setup}

(a)

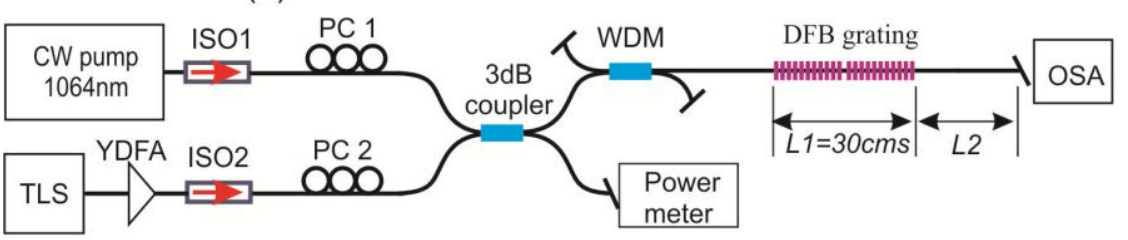

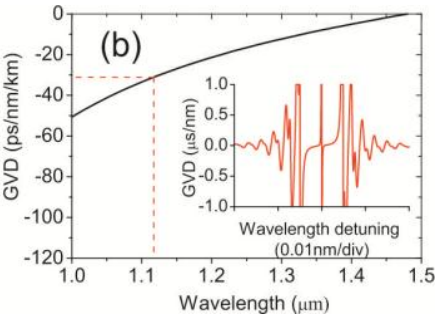

Fig. 1 (a) Schematic diagram of the experimental setup. TLS: tunable laser source; YDFA: Yb-doped fiber amplifier; ISO: isolator; PC: polarization controller; WDM: wavelength division multiplexer; OSA: optical spectrum analyzer. (b) Calculated total group-velocitydispersion (GVD) of PS980 and the inset is the calculated GVD of transmitted light of the DFB grating.

The schematic diagram of the experimental setup is shown in Fig. 1(a). A high power continuous-wave (CW) linearly polarized pump source at $\sim 1064 \mathrm{~nm}$ was used as the pump source for generating the R-DFB signal [7] 
and a TLS (DL pro, Toptica Photonics) was employed as the probe for FWM generation and was amplified by a PM Yb-doped fiber amplifier (YDFA). The outputs of the lasers were respectively connected to isolator1 (ISO1) and ISO2 and then polarization controller1 (PC1) and PC2. The $1064 \mathrm{~nm}$ pump source and the TLS were coupled into the DFB grating via a $1 \mu \mathrm{m} 3 \mathrm{~dB}$ coupler. Besides, a 1064/1117 nm WDM was inserted between the $3 \mathrm{~dB}$ coupler and the DFB grating in order to filter out the backward R-DFB signal. All the fiber ends were angle-cleaved to prevent end-feedback and the DFB grating was mounted on a heat sink to help control the temperature and better remove any generated heat. Note that all the passive components as seen in Fig. 1(a) were non-PM and all the components as well as the pigtail fiber were mounted statically on the optical bench to reduce the influence of the environmental fluctuations.

The centre $\pi$ phase-shifted DFB grating was written directly into PS980 fiber using $244 \mathrm{~nm}$ UV light inhouse. The coupling coefficient of the grating was measured to be $37 \mathrm{~m}^{-1}$ [8]. The same PS980 fiber was used as the pigtail of DFB grating with a length of $1.8 \mathrm{~m}$ (as L2 in Fig. 1(a)), which was directly coupled into an optical spectrum analyzer (OSA) via a free-space wavelength independent attenuator. The NA, propagation loss $(\alpha)$ at $1.1 \mu \mathrm{m}$, and nonlinear coefficient ( $\gamma @ 1.1 \mu \mathrm{m}$ ) of PS980 is $0.14,20 \mathrm{~dB} / \mathrm{km}$ and $\sim 6(\mathrm{Wkm})^{-1}$, respectively.

\section{Results and discussions:}

Fig. 1(b) shows the calculated total group velocity dispersion (GVD) of PS980 and the vertical dashed line indicates the operating wavelength of the R-DFB signal. The ZDW of the fiber is estimated to be $\sim 1.48 \mu \mathrm{m}$ and therefore all the operation wavelengths in this work are located in the normal dispersion region of the fiber. The inset of Fig. 1(b) shows the simulated GVD of the passive DFB grating within the wavelength detuning range of $\pm 0.02 \mathrm{~nm}$. It is clearly seen that the GVD reaches very high values within the pass-band of the grating around the R-DFB wavelength [9]. As the data also shows the dispersion drops off quite rapidly for wavelengths outside the stop-band of the DFB grating.
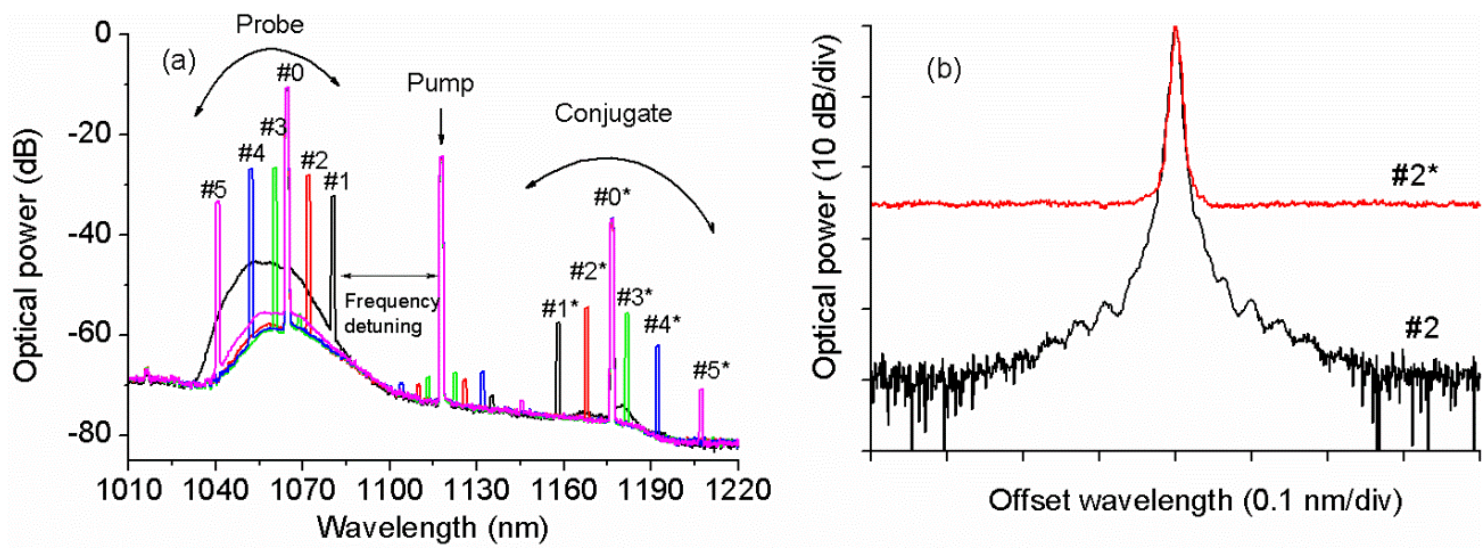

Fig. 2 (a) Typical FWM output spectra with a RBW of $1 \mathrm{~nm}$. (b) Normalized overlapping spectra of the probe wave \#2 (black) and its conjugate wave $\# 2 *$ (red) with a RBW of $0.01 \mathrm{~nm}$.

Fig. 2(a) shows the typical FWM output spectra with the TLS from the R-DFB device with a resolution bandwidth (RBW) of $1 \mathrm{~nm}$. Note that the R-DFB signal at $\sim 1117.8 \mathrm{~nm}$ was generated with the CW pump source at $1064 \mathrm{~nm}(\# 0)$ and then acted as the pump source for FWM process in the R-DFB device [6]. Also, the CW $1064 \mathrm{~nm}$ pump source was maintained constantly at $\sim 2 \mathrm{~W}$ [6] throughout the measurements of continuously tuning the TLS from $1080.4 \mathrm{~nm}(\# 1)$ to $\sim 1040.8 \mathrm{~nm}(\# 5)$. Such a CW 1064nm pump power was selected by means of ensuring the highest possible conversion efficiency of the conjugate wave $\# 0^{*}$ to its original wave \#0 without the TLS. At this power level, as clearly seen in Fig. 2(a), the conjugate waves from $\sim 1158 \mathrm{~nm}$ to $\sim 1207.3 \mathrm{~nm}$ clearly appear above the noise floor. For example, the conjugate wave of $\# \mathrm{i}^{*}(\mathrm{i}=0,1,2,3,4,5)$ is individually converted from its original probe wave of \#i via the FWM process. To further confirm this, Fig. 2(b) shows the normalized overlapping spectra of the probe wave of \#2 and the corresponding conjugate wave of \#2* with a RBW of $0.01 \mathrm{~nm}$. It is evident that they are nearly identical within the $25 \mathrm{~dB}$ bandwidth, which is expected since the FWM process will preserve the phase and amplitude of the wavelength-converted probe signal. Note that the noise floor of the conjugate wave is only limited by the sensitivity of the OSA.

The wavelength tunable range of the TLS applied in this work was restricted by the output power of the PM YDFA, as seen in Fig. 1(a). The incident probe power into the R-DFB device was in the range of 10 to $70 \mathrm{~mW}$, estimated from the output OSA traces. Fig. 3(a) shows the FWM conversion efficiency with respected to the frequency detuning which is the frequency separation between the probe wave and the R-DFB signal, as indicated in Fig. 2(a). The vertical lines indicate the conversion efficiency at the probe wavelengths of \#i $(\mathrm{i}=0,1,2,3,4,5)$ as shown in Fig. 2(a). It is found that the FWM conversion efficiency decreases from $\sim-25 \mathrm{~dB}$ to $\sim-37.5 \mathrm{~dB}$ while increasing the frequency detuning from $\sim 9.3 \mathrm{THz}$ (corresponding to the probe wave at 
$1080.4 \mathrm{~nm}$ ) to $\sim 19.9 \mathrm{THz}$ (corresponding to the probe wave at $\sim 1040.8 \mathrm{~nm}$ ). This is due to the larger phasemismatch factor between the waves at a wider frequency detuning [2]. However, it can be noted that there are two dips occurring at the probe waves of near \#0 and \#4 as seen in Fig. 3(a). This is believed to be due to the slight reduction of the R-DFB signal power, which can be proved by the inset of Fig. 2(a). The reasons for such a reduction of the R-DFB signal could be attributed to the power variation of the CW $1064 \mathrm{~nm}$ pump source, the environmental fluctuations and the slight drifting of the polarization state, which could be eliminated by applying all-PM fiber configuration. Regarding the effects of the variation of the probe power, Fig. 3(b) illustrates the FWM conversion efficiency against the YDFA pump diode current at selected probe waves of \#i $(\mathrm{i}=1,2,3,4,5)$, which have been indicated in Fig. 2(a) and Fig.3(a). It can be seen clearly that the FWM conversion efficiency remains constantly within $\pm 0.7 \mathrm{~dB}$ fluctuation. Note that the vertical line indicated in Fig. 3(b) is the YDFA pump diode current applied to all the probe waves during the measurement. Hence, it can be concluded that the reduction of the conversion efficiency with the increase of the frequency detuning in Fig. 3(a) is predominantly due to the dispersion effect and the power variation of the R-DFB signal, but not because of the power variation of the probe waves.

Such efficient and compact FWM generation in R-DFB fiber lasers has been theoretically studied by solving the FWM coupled mode equations [10]. The simulation results match well with the experimental data and will be presented in detail at the conference.
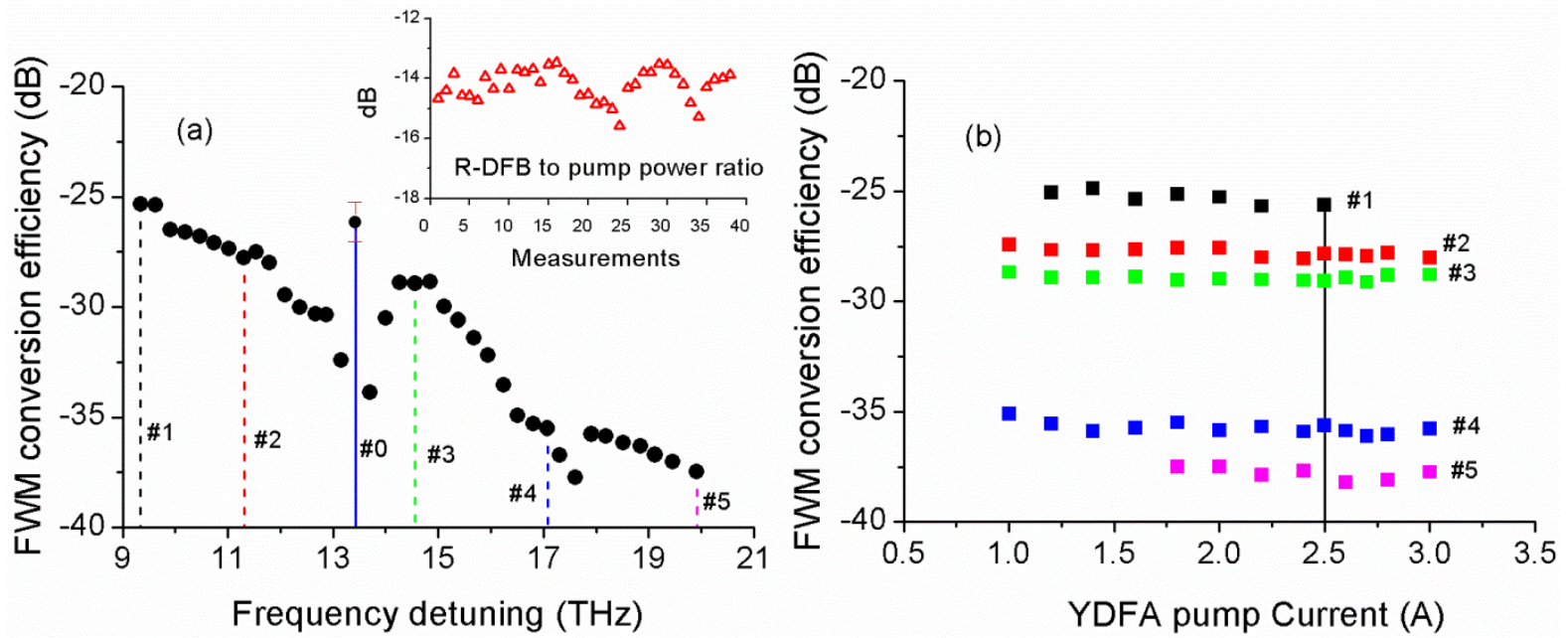

Fig. 3 (a) FWM conversion efficiency with respect to the frequency detuning and the inset is the R-DFB to its pump power ratio during the measurements. (b) FWM conversion efficiency vs YDFA pump diode current at selected probe wavelengths.

\section{Conclusion}

In summary, we have demonstrated ultra-broadband wavelength conversion based on FWM in a $30 \mathrm{~cm}$-long RDFB fiber laser in standard optical fiber. The conversion efficiency is measured to be up to $-25 \mathrm{~dB}$ over a wavelength conversion range of $\sim 167 \mathrm{~nm}$. To the best of our knowledge, this is the widest wavelength conversion with such a short length of a standard optical fiber.

\section{References:}

[1]. S. J. B. Yoo, "Wavelength conversion technologies for WDM network applications," J. of Lightwave Technol. 14, 955-966 (1996).

[2]. K. Inoue and H. Toba, "Wavelength conversion experiment using fiber four-wave mixing," Photon. Technol. Lett. 4, 69-72 (1992).

[3]. Z. Jianhui, P. Namkyoo, K. J. Vahala, M. A. Newkirk, and B. I. Miller, "Four-wave mixing wavelength conversion efficiency in semiconductor traveling-wave amplifiers measured to $65 \mathrm{~nm}$ of wavelength shift," Photon. Technol. Lett. 6, 984-987 (1994).

[4]. J. Minch, C. S. Chang, and S. L. Chuang, "Four-wave mixing in a distributed-feedback laser," Appl. Phys. Lett. 70, 1360-1362 (1997).

[5]. O. Aso, A. Shin-Ichi, T. Yagi, M. Tadakuma, Y. Suzuki, and S. Namiki, "Broadband four-wave mixing generation in short optical fibres," Electron. Lett. 36, 709-711 (2000).

[6]. J. Shi, S.-u. Alam, and M. Ibsen, "Ultra-Wide Range Wavelength Conversion Using FWM in a Raman DFB Fiber Laser," in Bragg Gratings, Photosensitivity, and Poling in Glass Waveguides (BGPP), BW3E.7 (2012).

[7]. J. Shi, S.-u. Alam, and M. Ibsen, "Sub-watt threshold, kilohertz-linewidth Raman distributed-feedback fiber laser," Opt. Lett. 37, 15441546 (2012).

[8]. J. Shi, S.-u. Alam, and M. Ibsen, "Highly efficient Raman distributed feedback fibre lasers," Opt. Expr. 20, 5082-5091 (2012).

[9]. Q. Zeng, "Dispersive properties in phase-shifted Bragg grating filters," in Antennas and Propagation Society International Symposium, 1062 1060-1063 (1998)

[10]. G. Agrawal, Nonlinear Fiber Optics, Third ed. (Springer Berlin / Heidelberg, 2000). 